\title{
Investigation of Composite Materials based on Modified Novolac Phenol-Formaldehyde Resin
}

\author{
Volodymyr Krasinskyi ${ }^{1, *}$, Oleh Suberlyak \\ 1 Lviv Polytechnic National University, Department of Chemical Technology of Plastics, 12, Bandera Str., Lviv, 79013, Ukraine
}

\begin{abstract}
It was determined that novolac phenol-formaldehyde resin modification by polyvinylpyrrolidone and epoxy-dianic resin results in a significant improvement of thixotropic characteristics and enamel technological effectiveness and in physicomechanical properties and thermostability improvement as well. The developed modified composition is characterized by high index of thixotropy in contrast to phenol-formaldehyde resin containing urotropine.
\end{abstract}

Keywords: novolac phenol-formaldehyde resin; polyvinylpyrrolidone; epoxy-dianic resin; modification; physico-mechanical properties; thixotropic characteristics; adhesion strength.

\section{Introduction}

In view of the extensive application of phenol-formaldehyde resins (PFR) in industry and private life, it is now necessary to develop new types of phenoplasts with high physicomechanical characteristics and a universal complex of properties for operation under various conditions. The universal applicability of these materials is connected with the broad temperature range of their hardening and the possibility of getting various operating characteristics depending on the field of their application.

The phenol-formaldehyde polymers are used as glue materials, anticorrosion coatings, and binders in the production of molding powders. The development of the field of polymeric glues and protective coatings requires the creation of new polymeric materials with given combinations of properties and, in the first place, with elevated adhesion strength and high water, chemical, and thermal resistances. The phenol-formaldehyde resins are extensively used for the purposes of gluing of glass with metals. Thus, the novolac PFR are used in the production of incandescent lamps as binders of the glue mastic aimed at connecting the metal base with the glass bulb [1]. Parallel with significant advantages (accessibility of raw materials, low-cost production, easiness of preparation of the lacquer, good dielectric properties, and high chemical stability), glue compositions based on the novolac PFR also have serious shortcomings: high brittleness of the filled PFR caused by their porosity and insufficient adhesion to metals and glass [2]. Therefore, the problem of development of new polymeric glue compositions and modification of the existing compositions used for gluing metals with glass always remains quite urgent [3].

As a possible direction of optimization of the properties of polymeric materials, we can mention their modification, i.e., controlled changes in their chemical compositions and physical structures. This can be realized by using both a great structural variety of the existing hardening agents for the polymeric materials and easiness of their chemical modification due to the presence of reactive groups [4]. 
The modification of phenol-formaldehyde compositions performed by using epoxy-dianic resins and polymers of the $\mathrm{N}$-vinyl series guarantees the possibility of complex improvement of the properties of "cross-linked" phenoplasts via the formation of additional three-dimensional networks in the resite and owing to the presence of polar functional groups in the macro chain. Therefore the new modification method is very important, in which resite elasticity and its adhesion to metals increase and high thermal stability is preserved. The decision of the problem is crosslinking of novolac PFR by ED-20 epoxy-dianic resin and polyvinylpyrrolidone (PVP) [5 excluding urotropine as a hardener because it forms a great amount of poison volatiles during resin hardening. The aim of this work is to develop thermostable reactive compositions with improved adhesive properties on the basis of phenol-formaldehyde resins of novolac type which are modified by polyvinylpyrrolidone and epoxy resin. The other aim is to establish the effect of modifiers on the technological and operational properties of obtained paintwork materials.

\section{Experimental Section}

The polymeric compositions were prepared in the following way. Phenol-formaldehyde lacquer (PFL) was obtained via dilution at $40-50^{\circ} \mathrm{C}$ of given mass of novolac phenol-formaldehyde resin in isopropyl alcohol. PVP was dried for $4 \mathrm{~h}$ at $60^{\circ} \mathrm{C}$ in an oven. The corresponding ass of PVP were dissolved in isopropyl alcohol and thoroughly mixed with PFL and epoxy resin. Then a catalyst for $\mathrm{N}, \mathrm{N}$-dimethylaniline (DMA) hardening was added. The surface hardness of polymeric material was determined using Heppler consistometer by indentation of steel cone with $53^{\circ} 08^{\prime}$ in the sample at the loading of $50 \mathrm{~N}$ for $60 \mathrm{~s}$. Impact elasticity was determined in accordance with GOST 464780 using pendulum hammer of 2083KM-0.4 type. Adhesion strength of glue line at uniform tearing and shearing were performed on a TIRA-test 2200 tensile-testing machine (Germany) with an extension rate of $50 \mathrm{~mm} / \mathrm{min}$.

\section{Results and Discussion}

The predicted change of physico-mechanical properties, PFR adhesive activity namely, is caused by controlled process of chemical interaction between PVP+PFR and epoxy resin ED-20 or PFR and ED-20 rather than physical effects due to the unique properties of PVP. Since the resin hardening takes place at high temperatures $\left(150-180^{\circ} \mathrm{C}\right)$ taking into account exoeffect, we may expect the reaction between free hydroxy groups of PFR and lactam cycles of PVP. These predictions are confirmed by a series of physical and chemical investigation methods - Differential Thermal Analysis (DTA), IR-spectroscopy, chemical titration, etc. [6].

The described factors essentially affect the physico-mechanical and adhesive properties of the composite. The increase of epoxy resin and DMA amount in the composition improves physicomechanical characteristics. Impact elasticity and surface hardness increase with the increase of ED-20 concentration to 25-30 mass \% (Fig. 1a) and DMA concentration - to 0.5-1 mass \% (Fig. 2). The physico-mechanical indices increase with the increase of hardening temperature.

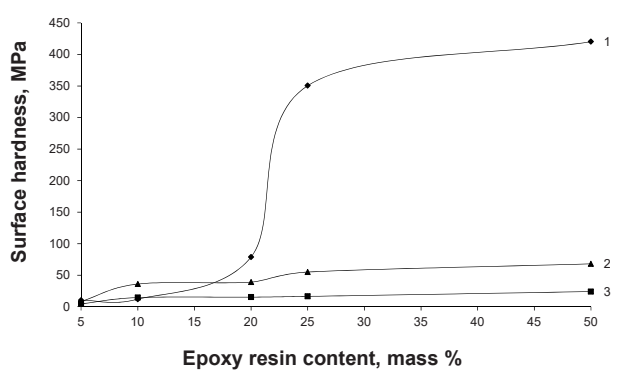

(a) *PVP content -0.5 mass $\%$, DMA -1 mass $\%$

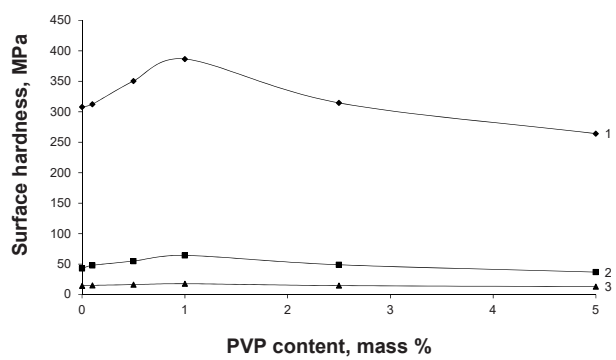

(b) *DMA - 1 mass $\%$, ED-20 - 25 mass $\%$

Fig. 1: Effect of ED-20 (a) and PVP (b) content on surface hardness of the compositions* depending upon hardening regime: $150-$ $160^{\circ} \mathrm{C}(1) ; 70-90^{\circ} \mathrm{C}(2)$ and $18-20^{\circ} \mathrm{C}(3)$

The dependence of physico-mechanical properties on PVP content has an extremal 
character with maximum at PVP content of 1 mass $\%$. The increase of PVP content higher than 1 mass $\%$ decreases thermal stability, impact elasticity and surface hardness (Figs. 1b, 3) independently on hardening regime.

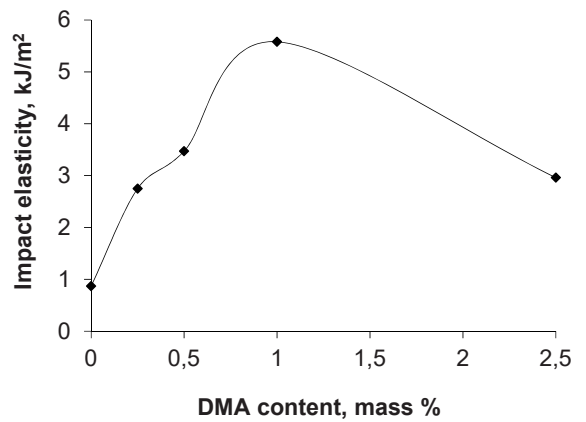

Fig. 2: Effect of DMA content on impact elasticity of phenolformaldehyde compositions* hardened at $150-160^{\circ} \mathrm{C}$ : *PVP content -0.5 mass \%, ED-20 - 25 mass \%.

One of the most important factors ensuring the strength of glued connections is adhesion strength and stability during operation. To determine the optimum ratio between composition components and hardening catalyst the samples were tested for adhesion strength of glue line at uniform tearing and shearing.

The effect of PVP content on adhesion has an extreme character. The adhesion has maximum value at PVP content up to 1.0 mass \%, then the decrease of glued connection strength is observed (Table 1). The values of adhesion strength are in a good agreement with concentrations of internal stress in glue lines.

We established that adhesion strength is maximum at ED-20: novolac PFR $=1: 3$ at DMA content of 1 mass \% (Table 1).

The insulating ability is an important characteristic of anticorrosive properties of coatings. Water is the most active reagent which easy penetrate into coatings. While staying of polymeric wares, namely films, in aqueous medium the adsorption of medium on polymer surface, its chemical interaction with undarable bonds, washing out and dilution of unbound and low-molecular compounds take place. But we synthesized the compositions with optimum ratio of components ensuring good physico-mechanical properties and low values of sorption ability in water. Such compositions may be used as a basis of anticorrosive coatings.

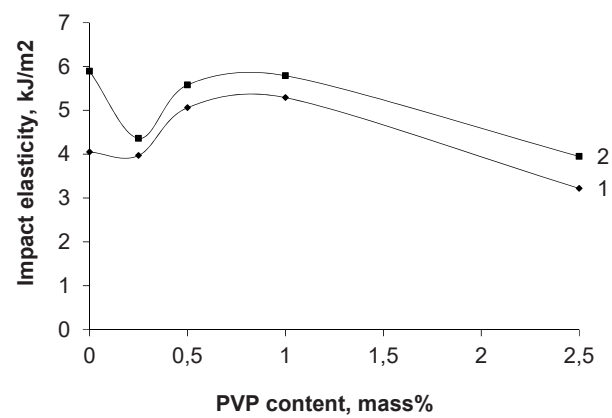

Fig. 3: Effect of PVP content on impact elasticity of phenolformaldehyde compositions hardened at $150-160^{\circ} \mathrm{C}$ : without ED20 at urotropine content of 4 mass \% (1) and ED-20 - 25 mass \%, DMA - 1 mass \% (2).

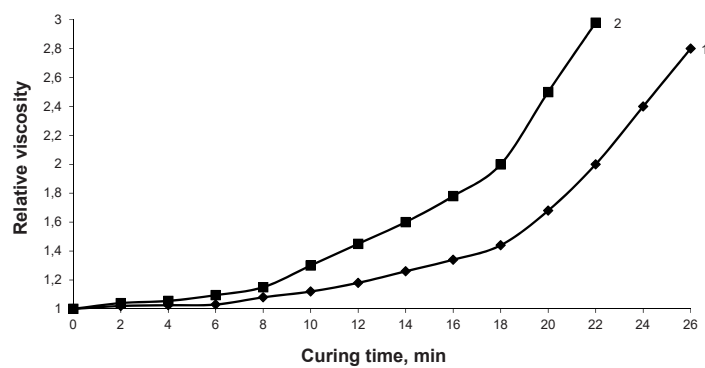

Fig. 4: Effect of PVP content on impact elasticity of phenolformaldehyde compositions hardened at $150-160^{\circ} \mathrm{C}$ : without ED20 at urotropine content of 4 mass \% (1) and ED-20 - 25 mass \%, DMA - 1 mass \% (2).

As far as synthesized compositions should be hardened at high temperatures $\left(150-160^{\circ} \mathrm{C}\right.$ ) to achieve excellent physico-mechanical and adhesive properties, the viscosity change during compositions hardening is of great importance. Fig. 4 represents the kinetic curves of viscosity change during phenol-formaldehyde compositions hardening. The kinetic curve has three areas with different character of viscosity exchange. For the first 8 minutes the viscosity is practically constant. In the second area (8-18 minutes) the viscosity increases with constant rate. In the third area (18-22 min. for curve 2 and 18-26 min. for curve 1) the viscosity changes with accelerated rate leading to the loss of material fluidity. The rate of viscosity increase is higher for the modified composition (curve 2) than that for non-modified PFR. Hence, the hardening time of modified composition is less for modified composition.

Since compositions based on PFR are widely used for the production of glues and various coatings, 
Tab. 1: Adhesion strength of glued connections "metal-glass" based on modified compositions depending on composition structure*

\begin{tabular}{|l|l|l|l|l|l|}
\hline \multicolumn{3}{|l|}{ Composition structure, mass \% } & $\begin{array}{l}\text { Adhesion shearing } \\
\text { strength, MPa }\end{array}$ & $\begin{array}{l}\text { Adhesion tearing } \\
\text { strength, MPa }\end{array}$ \\
\hline PFR & ED-20 & PVP & DMA & & 0.844 \\
\hline 74.25 & 25 & 0.5 & 0.25 & 1.962 & 1.356 \\
\hline 74 & 25 & 0.5 & 0.5 & 3.825 & 1.960 \\
\hline 73.5 & 25 & 0.5 & 1.0 & 5.756 & 3.252 \\
\hline 72 & 25 & 0.5 & 2.5 & 6.613 & \\
\hline & & & & & 1.329 \\
\hline 74 & 25 & 0 & 1 & 4.878 & 1.960 \\
\hline 73.5 & 25 & 0.5 & 1 & 5.756 & 2.385 \\
\hline 73 & 25 & 1.0 & 1 & 6.428 & 1.425 \\
\hline 71.5 & 25 & 2.5 & 1 & 4.839 & 1.087 \\
\hline 69 & 25 & 5.0 & 1 & 3.116 & \\
\hline & & & & & 0.428 \\
\hline 97.5 & 1 & 0.5 & 1 & 0.933 & 0.684 \\
\hline 93.5 & 5 & 0.5 & 1 & 1.612 & 0.952 \\
\hline 88.5 & 10 & 0.5 & 1 & 2.823 & 1.960 \\
\hline 73.5 & 25 & 0.5 & 1 & 5.756 & 2.114 \\
\hline 48.5 & 50 & 0.5 & 1 & 7.532 & \\
\hline
\end{tabular}

the development of composition with improved thixotropic properties is very urgent problem.

Fluidity curves for the developed composition are represented in Fig. 5. The reversion of structure is very quick, i.e. curves 1 and 2 obtained at the increase of shearing stress and the decrease of loading, relatively, are coincide with each other. Such composition is characterized by high value of thixotropy in contrast to PFR. The thixotropic structures are formed due to the physico-chemical interaction between active functional groups of phenol-formaldehyde oligomer and active functional groups of ED-20 and PVP forming the physical network. However such interaction does not affect viability of developed compositions which is sufficiently high (30 days at room temperature).

It should be noted that compositions with PVP are characterized by less value of shrinkage (Fig. 6) compared with compositions without PVP, though their hardening kinetics (according to viscosity change) is higher. This fact indicates the higher reactivity of binding agent.

The obtained results allow to establish the rational hardening time $\left(25-30 \mathrm{~min}\right.$.) at $150-160^{\circ} \mathrm{C}$. At the same time we proved that the composition may exist without changes at high (to $80^{\circ} \mathrm{C}$ ) temperatures.

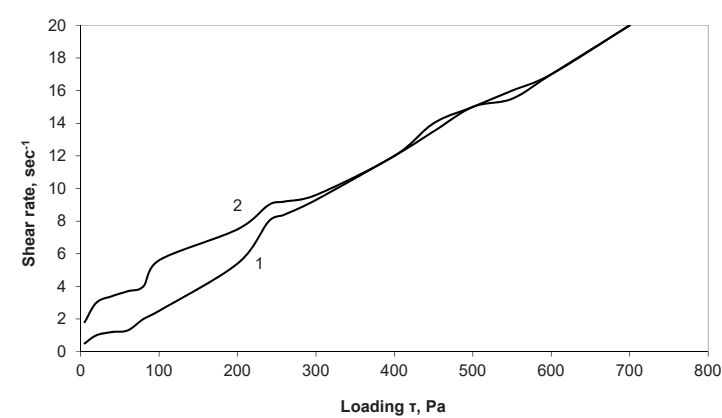

Fig. 5: Fluidity curves of PFR:ED-20:PVP:DMA $=73.5: 25: 0.5: 1$ composition: equilibrium curve (1) and non-equilibrium curve (2).

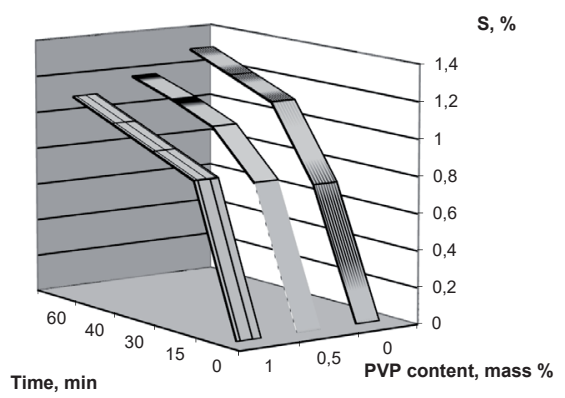

Fig. 6: Change ofshrinkage(S) during epoxy-novolaccompositions* hardening depending on PVP content and hardening time. * ED-20 -25 mass \%, DMA - 1 mass \%.

\section{Conclusions}

This research confirm the possibility of chemical modification of novolac phenol-formaldehyde resins by polyvinylpyrrolidone and epoxy resin at the temperatures higher than $120^{\circ} \mathrm{C}$. The modification of novolac PFR by polyvinylpyrrolidone and epoxy resin create conditions for the composition curing without urotropine. Owing to this fact we obtained thermostable non-toxic paintwork materials with high technological effectiveness and adhesion, good physico-mechanical properties and low water absorbing.

\section{References and Notes}

[1] Zaitseva, N. and Rodzivilova, I. (2003). Adsorption of PhenolFormaldehyde Oligomer on the Surface of Magnetic Fillers. Plast. Massy, 1, 19-23.

[2] Kuznetsova, 0., Arhireev, V. and Baranov, Yu. (2005). Hydroinsulating Compositions Based on Phenol-Formaldehyde Oligomers. Plast. Massy, 14, 31-34.

[3] Z. A. Kochnova, Z., Tuzova, S., Baranov, A. and Prut, E. (2002). Investigation of the structure and physicomechanical properties of the coatings based on epoxyphenol compositions. Plast. 
Massy, 8, 11-12.

[4] G. M. Tseitlin, G. and M. V. Zelenskaya, M. (1993). Specific features of the synthesis of novolac-epoxy oligomers. Lakokras. Mater., 5, 8-10.

[5] Suberlyak, 0., Shapoval, I. and Krasins'kyi, V. A Polymeric Composition Aimed at Gluing Steel with Glass [in Ukrainian], Patent of Ukraine No. 82171, MPK 08 5/09, 08 L 61/00, Publ. on 11.03.2008, Bull. No. 5.

[6] Suberlyak, 0., Krasins'kyi, V., Kochubei, V. and Shapoval, I. (2009). Influence of the chemical modification with polyvinylpyrrolidone on the physicomechanical properties of phenol-formaldehyde resin. Dopov. Nats. Akad. Nauk Ukr., 2, 148-153.

\section{Biographical notes}

Volodymyr Krasinskyi, Assoc. Professor, Ph.D. (born in 1982) is a lecturer at the Lviv Polytechnic National University, Department of Chemical Technology and Plastics Processing. He graduated at 2005 at the Lviv Polytechnic National University. His research activities are focused on the nanocomposites on the basis of thermoplastics and modified montmorillonite and on the heat-resistant coatings. He is an author of the 22 articles in international databases and of the 32 articles in the national database; number ORCID: http://orcid.org/0000-00016374-9340.

Oleh Suberlyak, Professor, Ph.D, D.Sc., (born in 1947) is a head of department and professor at the Lviv Polytechnic National University, Department of Chemical Technology and Plastics Processing. He graduated at 1969 at the Lviv Polytechnic National University. The most remarkable professional or academic positions is Honored Scientist of Ukraine. His research area is the development of technologies of obtaining and processing of polymers of a special purpose. He is an academician of the Technology Academy of Ukraine. He is an author of the 38 articles in international databases and of the 214 articles in the national database; number ORCID: http://orcid.org/0000-0002-6046-5972.

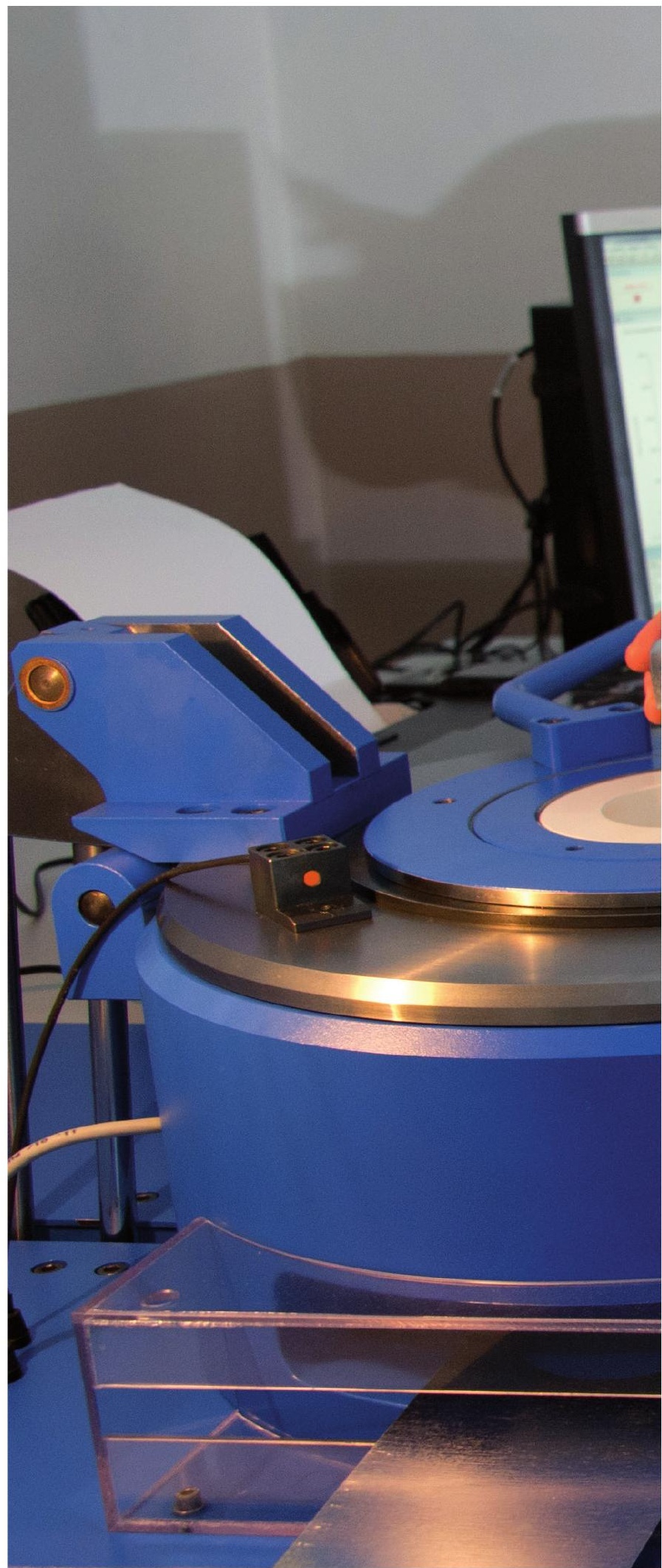

